\title{
Ternary Constituents Are a Consequence of Mora Sluicing
}

\author{
Ben Hermans and Francesc Torres-Tamarit \\ Meertens Institute and VU University Amsterdam
}

\section{Introduction}

Ternary stress is a unique challenge to constraint-based metrical stress theories: how to model ternarity without ternary feet (Halle \& Vergnaud 1987; Levin 1988; Dresher \& Lahiri 1991; Rice 1992) or ternaryspecific constraints that prohibit adjacent feet (Kager 1994; Houghton 2008).

While ternary rhythm exists, we propose that ternary feet do not, not even indirectly by means of recursion (pace Martínez-Paricio 2012; Kager \& Martínez-Paricio 2013a,b). We further propose that ternary rhythm arises from mora sluicing (Prince 1985; Hyde 2001), the phenomenon whereby a mora can be excluded from projecting to the 'syllable' level to satisfy an instance of the independently-motivated constraint No-CLASH (pace Elenbaas \& Kager 1999).

This paper is organized as follows. In $\S 2$, our representational assumptions are presented. In $\S 3$, we argue for mora sluicing based on evidence from uneven trochee-favoring processes in Mohawk and Central Slovak, and opaque stress-epenthesis interactions in Mohawk. The same rationale lies behind ternary stress systems, analyzed in $\S 4$. $\S 5$ concludes.

\section{Representational assumptions}

Our representations are based on Hammond 1984's Lollipop model, in which prominent positions in each prosodic plane have constituent status, and where every constituent is headed.

In the model developed here, moras are right-headed (Hyman 1985) (1). Moreover, 'codas' always head their own mora.

(1) Segments and moras<smiles>C[Al]1CC[Al]1C</smiles>

These moras may or may not project into what we call a line 1 constituent. In the first representation after the arrow in (2), only the vowel-headed mora projects up into a line1 constituent, whereas the consonantheaded mora shows mora sluicing, and therefore becomes a line1 dependent. This is not the case in the second representation after the arrow in (2), without mora sluicing.

(2) Moras and prominence

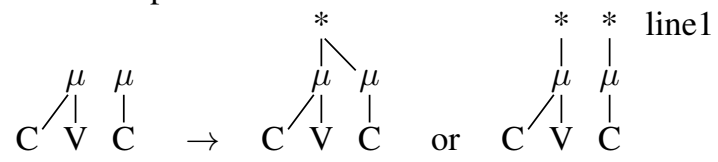

This model replaces syllables by line 1 constituents, and this move will be central for the analyses. For now, notice that line 1 constituents remotely correspond to syllables, in that a CVC sequence might be parsed by two, and not one, line 1 constituents when mora sluicing does not apply. When mora sluicing applies, it prevents a consonant-headed mora to project into the line1 level.

* We thank the audience of Phonology 2013 at the University of Massachusetts, Amherst, for their valuable comments. All errors and incongruities are, of course, ours alone.

(C) 2014 Ben Hermans and Francesc Torres-Tamarit

Proceedings of Phonology 2013

Completed January 27, 2014 
These line 1 constituents are then parsed into what we call line 2 constituents, which correspond more or less to metrical feet (3). These line 2 constituents are not binary-branching if mora sluicing applies, as can be seen in the first representation. We will use integers instead of more standard grid marks for expository reasons.

(3) (Left-headed) feet

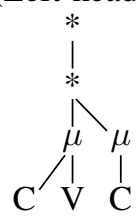

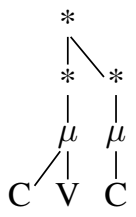<smiles>CNI(C)C</smiles>

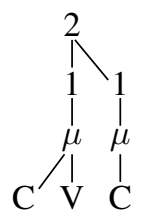

These ideas are not new. As said, they can be traced back in the works of Hammond (1984) and also Halle \& Vergnaud (1987), in which prominence and constituency are conflated, and constituents at any level are maximally binary branching, with an obligatory head and an optional dependent. What is new in our proposal is that even line 1 constituents conflate prominence with constituency. This fact is responsible for creating clashes at line1 if mora sluicing does not apply (4). If mora sluicing does apply, a clash at line1 is avoided (5).

(4) Clash at line1<smiles>C[Al](I)I(C)I</smiles>

(5) No clash at line1 due to mora sluicing<smiles>CC1C(I)[Al]1C</smiles>

The application or blocking of mora sluicing is driven by the ranking of two constraints: No-CLASH(1) (6) and $\operatorname{PROJECT} \mu$ (7).

(6) No-Clash(1)

Assign a violation mark for each pair of line1 constituents whose heads are adjacent.

(7) PRojeCt $\mu$

Assign a violation mark for every mora that does not project as the head of a line1 constituent.

If No-Clash(1) dominates Project $\mu$, we get mora sluicing. The opposite ranking thus blocks mora sluicing.

\section{Arguing for mora sluicing}

In this section, we argue in favor or mora sluicing in the light of data from Mohawk and Central Slovak, which have processes of vowel lengthening and vowel shortening, respectively. These processes seem to favor uneven trochees, those with a heavy head syllable and a light dependent syllable.

3.1 Mohawk In Mohawk, stress is penultimate. Additionally, if the stressed penultimate syllable is open, the stressed vowel lengthens (8a). However, if the stressed syllable is closed by a consonant, vowel lengthening is blocked (8b-c). 
(8) Penultimate stress in Mohawk (Mellander 2003)
(a) /wak-haratat-u/ wakhara('ta:tu)
'I am holding it up'
(b) /wak-haratat-u-hatye- $\emptyset / \quad$ wakharatatu('hatye) 'I go along lifting up'
(c) /k-atirut-ha/ kati('rutha) 'I pull'

Independently, inflected verb forms are subject to a disyllabic word-minimality requirement. Monovocalic underlying forms are repaired via word-initial vowel epenthesis. The interesting thing is that the epenthetic vowel also lengthens if the newly created syllable is open ( $9 b$ as opposed to $9 a$ ).

(9) Word-initial vowel epenthesis
(a) $/ \mathrm{k}-\mathrm{k} \Lambda-\mathrm{s} /$
'I see'
(b) /w-e-?s/
('ik.kıs)
('izwe?s)
'She/it is walking around'

We take this process of open syllable vowel lengthening as an argument in favor of the uneven trochee (HL), as in Mellander 2003 (pace Hayes 1989). We further interpret open syllable vowel lengthening as a way to satisfy No-CLASH(1) via an operation of mora insertion plus mora sluicing (10).

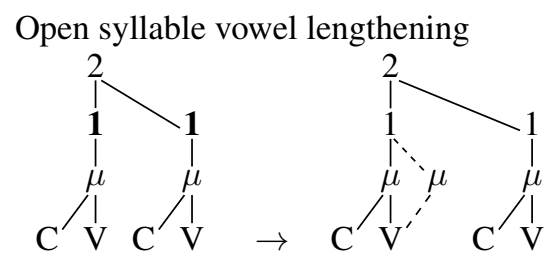

This mapping obtains from ranking No-CLASH(1) above PROJECT $\mu$ and DEP $\mu$. One argument in favor of considering open syllable vowel lengthening as a phonological, and not phonetic, process comes from an opaque stress-epenthesis interaction. If an epenthetic vowel is required in a penultimate syllable to break a consonantal cluster, stress is antepenultimate and the stressed vowel is not lengthened (11).

(11) Epenthesis in penultimate position and stress retraction
(a) / $/ \Lambda-k-r-\Lambda P / \quad$ ' $k e r \Lambda ?$
'I will put it into a container'
(b) /te-k-rik-s/ 'tekeriks 'I put them next to each other'
cf. (c) /wak-ashet-u/ wakas'he:tu
'I have counted it'

These cases of stress retraction can also be interpreted as emerging from structures where mora sluicing has applied. However, in opaque stress-epenthesis interactions of this type, mora sluicing affects a whole syllable, the one containing the epenthetic vowel (italicized in 12).

(12) Mora sluicing over a whole syllable containing an epenthetic vowel

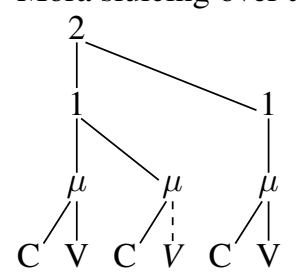

Mora sluicing over a syllable whose nucleus is an epenthetic vowel is favored by a constraint penalizing non-input segments to head a line1 constituent. This constraint is analogous to Alderete (1999)'s HEADDEP 
constraint. HEAdDEP also dominates PROJECT $\mu$, and this ranking is responsible for creating a ternary constituent. ${ }^{1}$

We have shown that both open syllable vowel lengthening and stress retraction in Mohawk can be explained in unison by means of mora sluicing.

3.2 Central Slovak Data from Central Slovak also offers evidence in favor of the uneven trochee. Underlying long vowels shorten after stressed long vowels (13a). However, unstressed long vowels are not shortened after stressed short vowels (13b).

(13) Post-tonic vowel shortening after long vowels (Mellander 2003)
(a) /ba:s-ji:k/ ('ba:snik)
'poet'
(b) /les-ni:k/ ('lesni:k)
'forester'

We interpret this process of post-tonic vowel shortening after long vowels as a way to satisfy a branchingness condition on line 2 dependent constituents.

Apart from No-CLASh(1) and ProjeCt $\mu$, we also propose a set of constraints that impose branchingness conditions on metrical constituents. They are of two types, those that refer to heads and those that refer to dependents. The former disfavor non-branching heads, and the latter disfavor branching dependents (14). The idea behind these constraints is that there is an asymmetry in the amount of complexity allowed in head and dependent positions (Dresher \& van der Hulst 1998).

(14) Branchingness constraints

(a) The head of constituent $C$ must branch

(b) The dependent of constituent $C$ must not branch

One instance of this family of constraints, call it LINE2Dep $\rightarrow \mid$ (15), is what triggers post-tonic vowel shortening if ranked above the anti-shortening faithfulness constraint $\mathrm{MAX} \mu$.

\section{(15) LiNE2Dep $\rightarrow \mid$}

Assign a violation mark for every line2 dependent constituent that branches.

In (16), the dependent of the line 2 constituent, that is, the second line 1 constituent, branches. This representation violates the constraint LINE2Dep $\rightarrow \mid$, and therefore maps into a representation where the dependent of the second line 1 constituent, the second mora, deletes. ${ }^{2}$

Vowel shortening

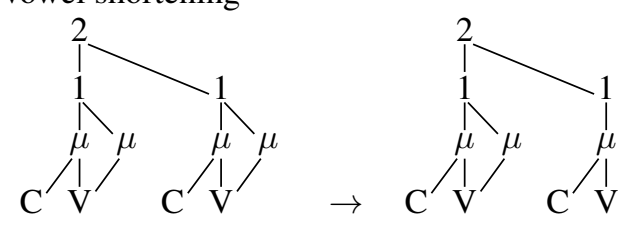

\footnotetext{
${ }_{1}^{1}$ Stress retraction causing ternary rhythm in Mohawk is restrained to cases where the penultimate vowel is epenthetic. In contradistinction to this, the optimal parsing of an input like /CVCVCV/, with three underlying vowels, results in penultimate stress and open syllable vowel lengthening. However, in a genuine ternary stress language, as will be shown in the next section, /CVCVCV/ is mapped with antepenultimate stress. The fact that in Mohawk /CVCVCV/ surfaces with penultimate stress derives from ranking LINE1Dep $\rightarrow \mid$ above DEP $\mu$. The constraint LINE1Dep $\rightarrow \mid$ is violated when a CV sequence, parsed into a branching mora, is a line 1 dependent. Ternary stress is only derived from /CVCCV/ inputs because $* \mathrm{CC}$, a constraint against consonantal clusters, dominates LINE1Dep $\rightarrow \mid$. In a genuine ternary stress language, however, DEP $\mu$ dominates LiNE1Dep $\rightarrow \mid$. In both languages, No-Clash(1), the constraint triggering either vowel lengthening or ternarity, dominates PROJECT $\mu$.

2 The constraint LINE2Dep $\rightarrow \mid$ is also violated when the post-tonic syllable is closed by a consonant, because a CVC sequence always projects two moras in the proposed model. However, ranking MAXC above MAX $\mu$ is enough to block consonant deletion in this cases.
} 
As said, vowel shortening is blocked in post-tonic position if the stressed syllable contains a short vowel. The proposed branchingness constraint LINE2Dep $\rightarrow \mid$ also assigns a violation to the representation in (17), where the dependent of the line 2 constituent branches. In principle, the constraint LINE2Dep $\rightarrow \mid$ should also trigger vowel shortening in these cases, after a stressed short vowel. However, this is not the case. It is precisely here when mora sluicing plays a crucial role. We propose that the representation in (17) is actually harmonically-bounded by that in (18), where the first half of the unstressed long vowel, its first mora, is parsed as the dependent of the first line 1 constituent via mora sluicing. This way, the constraint LINE2Dep $\rightarrow \mid$, which is independently needed to explain post-tonic vowel shortening after long vowels, is satisfied.

(17) Non-optimal ('LH)

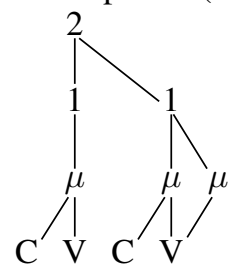

(18) Well-formed ('LH) with internal mora sluicing

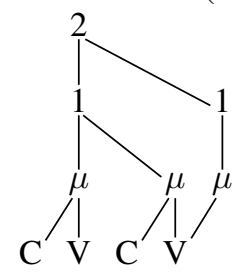

Summing up, the notion of mora sluicing has been argued to explain two different uneven trocheefavoring processes, namely open syllable vowel lengthening in Mohawk and post-tonic vowel shortening in Central Slovak. Mora sluicing involves a violation of the constraint PROJECT $\mu$, which demands that every mora is the head of a line 1 constituent. Mora sluicing is a strategy triggered by different markedness constraints, No-CLASH(1) and HEADDEP in the case of Mohawk and LINE2Dep $\rightarrow \mid$ in the case of Central Slovak. We have seen that mora sluicing creates ternary constituents at the mora level in both Mohawk and Central Slovak.

In the next section, we will argue that the same notion of mora sluicing is the responsible for ternary stress.

\section{Ternary stress is the consequence of mora sluicing}

4.1 Ternary non-exhaustive parsing systems: data Cayuvava and Tripura Bangla illustrate ternary non-exhaustive parsing systems (19). First consider (19a). In Cayuvava, stress falls on every third syllable from the right, with primary stress on the rightmost stressed syllable. This pattern has traditionally been described as assigning right-to-left trochees with final-syllable extrametricality. The forms in (19b) stand for Tripura Bangla, where primary stress falls on the first syllable and secondary stress on every third syllable afterwards except when this would create final stress. What Cayuvava and Tripura Bangla share is that up to two syllables are left unparsed at the opposite edge of footing. This is observed in five- and eight-syllable words in Cayuvava, and in four- and seven-syllable words in Tripura. 
(19) Cayuvava (Key 1967) and Tripura Bangla (Das 2001)

\begin{tabular}{|c|c|c|}
\hline & (a) Cayuvava & (b) Tripura Bangla \\
\hline $2 \sigma$ & $(' \sigma \sigma)$ & $(' \sigma \sigma)$ \\
\hline $3 \sigma$ & $(' \sigma \sigma) \sigma$ & $(' \sigma \sigma) \sigma$ \\
\hline $4 \sigma$ & $\sigma(' \sigma \sigma) \sigma$ & $(' \sigma \sigma) \sigma \sigma$ \\
\hline $5 \sigma$ & $\sigma \sigma\left({ }^{\prime} \sigma \sigma\right) \sigma$ & $(\sigma \sigma) \sigma(\sigma \sigma)$ \\
\hline $6 \sigma$ & $({ } \sigma \sigma) \sigma\left({ }^{\prime} \sigma \sigma\right) \sigma$ & $\left({ }^{\prime} \sigma \sigma\right) \sigma(\sigma \sigma) \sigma$ \\
\hline $7 \sigma$ & $\sigma\left({ }_{1} \sigma \sigma\right) \sigma\left({ }^{\prime} \sigma \sigma\right) \sigma$ & $\left({ }^{\prime} \sigma \sigma\right) \sigma(, \sigma \sigma) \sigma \sigma$ \\
\hline $8 \sigma$ & $\sigma \sigma(, \sigma \sigma) \sigma(' \sigma \sigma) \sigma$ & $(\sigma \sigma) \sigma(\sigma \sigma) \sigma(\sigma \sigma)$ \\
\hline $9 \sigma$ & $\left({ }_{1} \sigma \sigma\right) \sigma\left({ }_{1} \sigma \sigma\right) \sigma\left({ }^{\prime} \sigma \sigma\right) \sigma$ & $\left.{ }^{\prime} \sigma \sigma\right) \sigma(, \sigma \sigma) \sigma(, \sigma \sigma) \sigma$ \\
\hline
\end{tabular}

4.2 Ternary exhaustive parsing systems: data The second type of ternary languages are illustrated with data from Estonian and Chugach Alutiiq. First consider Estonian (20a). As in Tripura Bangla, trochees are assigned from left to right and final stress is prohibited. However, how final stress is avoided in Estonian differs from Tripura. In Tripura, on the one hand, two syllables are left unparsed in these cases, for instance in four-syllable words. In Estonian, on the other hand, the last secondary stress is only one syllable away from the previous secondary stress, thus mixing binary and ternary rhythm in $3 n+1$-syllable words. Chugach is like Estonian but it assigns iambs instead of trochees (20b).

(20) Estonian and Chugach Alutiiq (Elenbaas \& Kager 1999)

$\begin{array}{lll} & \text { (a) Estonian } & \text { (b) Chugach Alutiiq } \\ 2 \sigma & \left({ }^{\prime} \sigma \sigma\right) & \left(\sigma^{\prime} \sigma\right) \\ 3 \sigma & \left({ }^{\prime} \sigma \sigma\right) \sigma & \left(\sigma^{\prime} \sigma\right) \sigma \\ 4 \sigma & \left({ }^{\prime} \sigma \sigma\right)(, \sigma \sigma) & \left(\sigma^{\prime} \sigma\right)(\sigma, \sigma) \\ 5 \sigma & \left({ }^{\prime} \sigma \sigma\right) \sigma(\sigma \sigma) & \left(\sigma^{\prime} \sigma\right) \sigma(\sigma, \sigma) \\ 6 \sigma & \left({ }^{\prime} \sigma \sigma\right) \sigma(\sigma \sigma) \sigma & \left(\sigma^{\prime} \sigma\right) \sigma(\sigma, \sigma) \sigma \\ 7 \sigma & \left({ }^{\prime} \sigma \sigma\right) \sigma(\sigma \sigma)(, \sigma \sigma) & \left(\sigma^{\prime} \sigma\right) \sigma(\sigma, \sigma)(\sigma, \sigma) \\ 8 \sigma & \left({ }^{\prime} \sigma \sigma\right) \sigma(\sigma \sigma) \sigma(\sigma \sigma) & \left(\sigma^{\prime} \sigma\right) \sigma(\sigma, \sigma) \sigma(\sigma, \sigma) \\ 9 \sigma & \left({ }^{\prime} \sigma \sigma\right) \sigma(\sigma \sigma) \sigma(\sigma \sigma) \sigma & \left(\sigma^{\prime} \sigma\right) \sigma(\sigma, \sigma) \sigma(\sigma, \sigma) \sigma\end{array}$

\subsection{Analysis}

More on representations In our model, line 2 constituents roughly correspond to metrical feet, but main stress is also a constituent, as in the early days of metrical phonology (Liberman \& Prince 1977). We represent main stress as a line 3 constituent.

In (21), a schematic representation is illustrated, where each CV sequence projects as the head of a line 1 constituent, and the first two line 1 constituents are parsed as a left-headed line 2 constituent. This line 2 constituent is the head of the line 3 constituent, which takes as its dependent the last line 1 constituent.

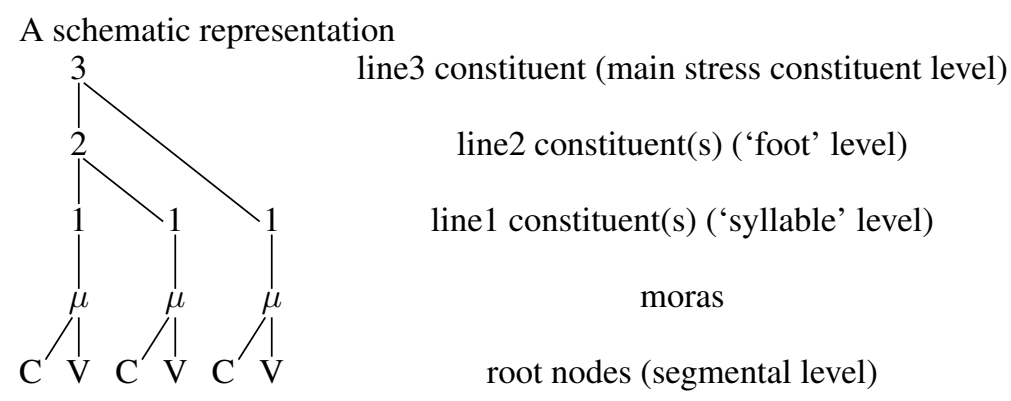

4.3.1 Binary vs. ternary rhythm Any analysis of ternary stress must account for (a) the difference between binarity and ternarity, and (b) the difference between non-exhaustive and exhaustive systems among ternary languages.

\footnotetext{
3 In this presentation we consider words containing only light syllables; weight effects will not be discussed.
} 
First we derive the difference between binary and ternary rhythm, which depends on how the already familiar constraints NO-CLASH(1) and PROJECT $\mu$ are ranked. Binarity obtains by ranking PROJECT $\mu$ above No-Clash(1). In the tableau in (22), candidates have six syllables each. On the one hand, candidate (a) parses each pair of $\mathrm{CV}$ sequences into three line 2 constituents, thus creating a binary rhythmic alternation. On the other hand, candidate (b) parses two sets of three CV sequences into two line 2 constituents, thus creating ternarity. Notice that all line 2 constituents are binary branching in both candidates, and therefore a constraint like FOOT-BINARITY, or a homologous constraint, is satisfied. The difference between candidate (a) and candidate (b) is whether mora sluicing applies or not. In candidate (a), each CV sequence corresponds to a line 1 constituent, whereas in candidate (b), some line 1 constituents cover two CV sequences. This fact allows for line 2 constituents to take as a dependent an extra CV sequence, thus creating ternary rhythm. Candidate (b) is ruled out because it fatally violates PROJECT $\mu$. This is why candidate (a), with binary rhythm, is more harmonic; it satisfies PROJECT $\mu$ at the expense of violating low-ranked NO-CLASH(1). The generalization behind this analysis is that binary rhythm results from the projection of every mora as the head of a line 1 constituent at the expense of clashes at the line1 level.

(22) Binary rhythm ${ }^{4}$

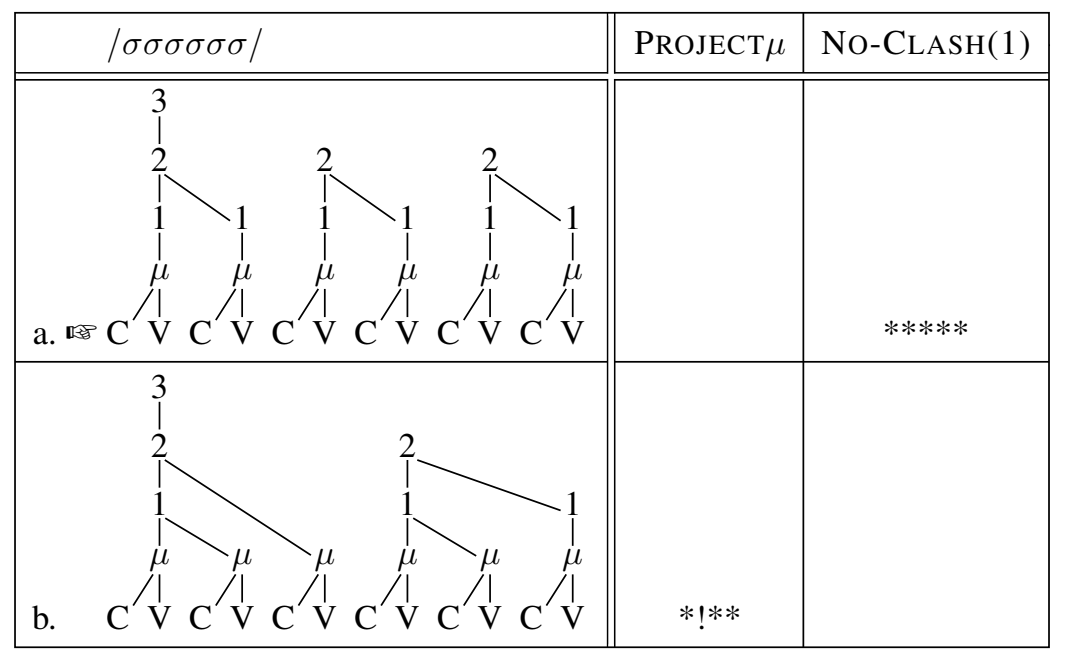

Ternary stress obtains with the opposite ranking, where NO-CLASH(1) dominates PROJECT $\mu$. In the tableau in (23), ranking No-CLASH(1) above PROJECT $\mu$ favors the candidate with ternary rhythm, in this case candidate (a), over the candidate showing binary rhythm, candidate (b).

\footnotetext{
4 We abstract away from directionality in this analysis, which is the result of the alignment constraints HEADFOOTRight/Left, which refers to line3, the main stress constituent, and ALLFEET-Right/Left, as well as from the foot type constraints TROCHEE and IAMB.
} 
(23) Ternary rhythm

\begin{tabular}{|c|c|c|}
\hline$|\sigma \sigma \sigma \sigma \sigma \sigma|$ & No-ClASH(1) & PROJECT $\mu$ \\
\hline 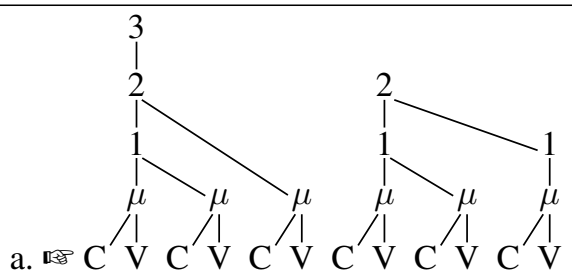 & & . \\
\hline 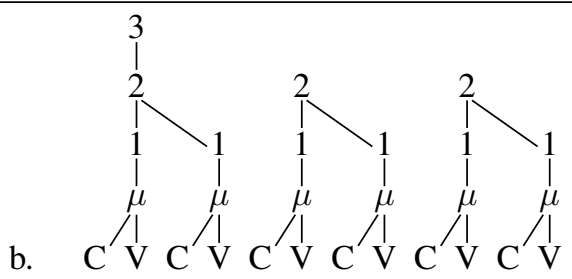 & $* ! * * * *$ & \\
\hline
\end{tabular}

4.3.2 Exhaustive vs. non-exhaustive ternary rhythm Among ternary stress systems, the most crucial difference is found in $3 n+1$-syllable words, where the difference between exhaustive and non-exhaustive parsing is detectable. In both types of ternary languages, final stress at the opposite edge of footing is prohibited because it would entail a degenerate, non-branching line 2 constituent. The way non-branching line2 constituents at the opposite edge of footing are avoided, however, differs between the two types of systems. In languages like Tripura Bangla, two CV sequences are left unparsed, whereas in languages like Estonian, ternarity is disrupted and binary rhythm obtains between the penultimate and last secondary stress. In the latter case, we call the pattern exhaustive.

The difference between these two systems depends on the ranking of two constraints not presented before. We call them PARSE $\mu$ (2) (24), which roughly corresponds to PARSE $\sigma$, and No-ClASH(2) (25).

(24) $\operatorname{PARSE} \mu(2)$

Assign a violation mark for every mora that is not parsed into a line 2 constituent.

(25) No-Clash(2)

Assign a violation mark for each pair of line 2 constituents whose heads are adjacent.

An exhaustive system like the one found in Estonian obtains from ranking PARSE $\mu(2)$ above NoClash(2). The tableau in (26) contains competitors with seven syllables each. After the main stress constituent, which parses the first three CV sequences, four moras are left. Candidate (a) parses two pairs of moras into two line 2 constituents. This allows for satisfying $\operatorname{PARSE} \mu(2)$ at the expense of violating NoCLASH(2), which is violated by binary rhythm in systems with mora sluicing, because the heads of the last two line 2 constituents are adjacent. However, candidate (b) does not violate No-CLASH(2) because it builds a second ternary line 2 constituent and leaves the last mora unparsed, thus incurring a fatal violation of $\operatorname{PARSE} \mu(2)$. A third candidate in which the last mora is parsed into its own line2 constituent, not included in this tableau, would be discarded by a top-ranked constraint requiring line 2 constituents to have at least two moras (as said before, a constraint analogous to FOOT-BINARITY). Exhaustive ternary rhythm thus results from parsing all moras into line 2 constituents at the expense of creating clashes at the line 2 level. 
(26)

Exhaustive ternary rhythm

\begin{tabular}{|c|c|c|}
\hline$|\sigma \sigma \sigma \sigma \sigma \sigma \sigma|$ & $\operatorname{PARSE} \mu(2)$ & No-Clash(2) \\
\hline$a$ & & $*$ \\
\hline b. & $* !$ & \\
\hline
\end{tabular}

The opposite ranking, that is, NO-CLASH(2) dominating PARSE $\mu(2)$, results in non-exhaustive ternary rhythm, like the one found in Tripura Bangla. In the tableau in (27), the candidate showing mora underparsing is more harmonic than the candidate with clashes at the line2 level.

Non-exhaustive ternary rhythm

\begin{tabular}{|c|c|c|}
\hline$|\sigma \sigma \sigma \sigma \sigma \sigma \sigma|$ & No-CLASH(2) & $\operatorname{PARSE} \mu(2)$ \\
\hline a. & & $*$ \\
\hline b. & $* !$ & \\
\hline
\end{tabular}

\section{Conclusions}

To conclude, the main claim of this paper is that ternary stress is a consequence of mora sluicing. The notion of mora sluicing has been proved to have high explanatory power: it is necessary on independent grounds to explain otherwise unrelated phenomena such as uneven trochee-favoring processes in Mohawk and Central Slovak, and opaque stress-epenthesis interactions in Mohawk (but also final-consonant extrametricality and three-syllable window systems, see Hermans 2013). One fundamental move derived from the metrical model sketched in this paper is the relation between what we call linel constituents (phonological syllables) and phonetic syllables (namely CV or CVC sequences), which is not one-to-one anymore, as it is in standard syllable theory.

We suggest that all necessary types of feet (including the uneven trochee) are derived from our model. Two phonetic syllables or one heavy phonetic syllable may correspond to one line1 constituent via mora sluicing. This would be the case of ternary stress or uneven trochees (28). 
(28) Ternary stress and uneven trochee<smiles>C[Al](C)[IH][In]I</smiles>

Moreover, one phonetic syllable may be parsed as one line1 constituent also via mora sluicing. This one-to-one relation would correspond to a syllabic trochee (29).

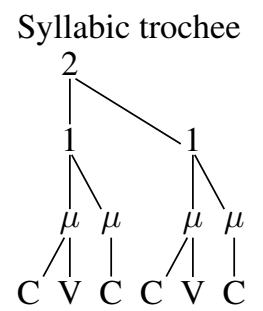

Finally, one phonetic syllable may be parsed into two line1 constituents, giving rise to a moraic trochee.

(30) Moraic trochee<smiles>C[Al]=IC=IC</smiles>

Our proposal accounts for ternary rhythm via mora sluicing without the need for postulating recursive feet (pace Martínez-Paricio 2012; Kager \& Martínez-Paricio 2013a,b). We think that the null hypothesis should be that prosodic constituents are not recursive unless they interface with syntax, the recursive component of grammar (Chomsky 2000). This way, prosodic words or phonological phrases may show recursion inherited from syntax (Elfner 2012). However, we claim that prosodic constituents below the prosodic word, for instance feet or line 2 constituents in our vocabulary, never show recursion. Our main objection against approaches of ternary stress based on recursive feet is that recursion is restrained to only one iteration, thus betraying the concept of recursion itself.

\section{References}

Alderete, John (1999). Head dependence in stress-epenthesis interaction. Hermans, Ben \& Marc van Oostendorp (eds.), The derivational residue in phonological Optimality Theory, Amsterdam: John Benjamins, 29-50.

Chomsky, Noam (2000). Minimalist inquiries: the framework. Roger Martin, David Michaels \& Juan Uriagereka (eds.), Step by step: Essays on minimalist syntax in honor of Howard Lasnik, Cambridge, MA: The MIT Press.

Das, Shyamal (2001). Some aspects of the prosodic phonology of Tripura Bangla and Tripura Bangla English. Ph.D. thesis, Central Institute of English and foreign languages, Hyderabad.

Dresher, B. Elan \& Harry van der Hulst (1998). Head-dependent asymmetries in phonology: complexity and visibility. Phonology 15, 317-352.

Dresher, Elan B. \& Aditi Lahiri (1991). The germanic foot: metrical coherence in Old English. Linguistic Inquiry :22, 251-286.

Elenbaas, Nine \& René Kager (1999). Ternary rhythm and the lapse constraint. Phonology 16, 273-329.

Elfner, Emily (2012). Syntax-prosody interactions in Irish. Ph.D. thesis, University of Massachusetts, Amherst.

Halle, Morris \& Jean-Roger Vergnaud (1987). An essay on stress. Dordrecht: Foris.

Hammond, Michael (1984). Constraining metrical theory: a modular theory of rhythm and destressing. Ph.D. thesis, University of California, Los Angeles.

Hayes, Bruce P. (1989). Compensatory lengthening in moraic phonology. Linguistic Inquiry 20, 253-306.

Hermans, Ben (2013). Conditions on branchingness. Talk presented at the 21st Manchester Phonology Meeting.

Houghton, Paula (2008). Positionally licensed extended lapses. University of Pennsylvania Working Papers in Linguistics 14, 201-212. 
Hyde, Brett (2001). Metrical and prosodic structure in Optimality Theory. Ph.D. thesis, Rutgers, The State University of New Jersey.

Hyman, Larry M. (1985). A theory of syllable weight. Dordrecht: Foris.

Kager, René (1994). Ternary rhythm in alignment theory. Ms. Utrecht University. Available on Rutgers Optimality Archive, ROA 35.

Kager, René \& Violeta Martínez-Paricio (2013a). Non-intervention constraints and the binary-to-ternary rhythmic continuum. Talk presented at the 21st Manchester Phonology Meeting.

Kager, René \& Violeta Martínez-Paricio (2013b). Ternary stress in parallel and serial OT: consequences for representations. Poster presented at Phonology 2013, University of Massachusetts.

Key, Harold (1967). Morphology of Cayuvava. The Hague: Mouton de Gruyter.

Levin, Juliette (1988). Generating ternary feet. Texas Linguistic Forum 29, 97-113.

Liberman, Mark \& Alan S. Prince (1977). On stress and linguistic rhythm. Linguistic Inquiry 8, 249-336.

Martínez-Paricio, Violeta (2012). Superfeet as recursion. Proceedings of the West Coast Conference on Formal Linguistics 30, 259-269.

Mellander, Evan W. (2003). (HL)-creating processes in a theory of foot structure. The Linguistic Review 20, $243-280$.

Prince, Alan S. (1985). Improving tree theory. Proceedings of the 11th Annual Meeting of the Berkeley Linguistics Society 471-490.

Rice, Curt (1992). Binarity and ternarity in metrical theory: parametric extension. Ph.D. thesis, University of Texas, Austin. 